\title{
Physical activity can improve cognition in patients with Alzheimer's disease: a systematic review and meta-analysis of randomized controlled trials
}

This article was published in the following Dove Press journal:

Clinical Interventions in Aging

\section{Zhen Du' \\ Yuewei $\mathrm{Li}^{\prime}$ \\ Jinwei Li' \\ Changli Zhou' \\ Feng $\mathrm{Li}^{1}$,* \\ Xige Yang ${ }^{2, *}$}

'Department of Internal Nursing, School of Nursing, Jilin University, Changchun, Jilin, I 30020, People's Republic of China; ${ }^{2}$ Department of Anesthesiology, The First Hospital of Jilin University, Changchun, Jilin, |3002 |, People's Republic of China

*These authors contributed equally to this work
Correspondence: Feng Li

Department of Internal Nursing,

School of Nursing, jilin University,

965 Xinjiang Street, Changchun, Jilin,

130020, People's Republic of China

Tel +86 I 7790089009

Fax +86043I856I 9580

Email fli@jlu.edu.cn

Xige Yang

Department of Anesthesiology,

The First Hospital of Jilin University,

7I Xinmin Street, Changchun, Jilin,

I3002 I, People's Republic of China

Tel +86 I $375666 \quad 628$

Fax +8604318 8782955

Email xige_yang@।63.com
Background/objective: Alzheimer's disease (AD) is mainly characterized by decline of cognitive functions such as memory and learning, which has a high prevalence and poor drug efficacy in treatment regimes. A systematic review and meta-analysis of randomized controlled trials (RCTs) were conducted to evaluate the effectiveness of exercise on cognitive function in patients diagnosed with AD.

Methods: The bibliographic databases (PubMed, Cochrane Library and Embase, and Web of Science) and four Chinese databases (Wanfang data, CBM, CNKI, and VIP) were searched to identify RCTs published in any language between January 1, 1960, and January 1, 2018. Only peer-reviewed articles and RCTs were included. The collected data were analyzed by Review Manager (5.3).

Results: Overall, 869 patients diagnosed with AD were included from 13 RCTs. Patients in the intervention group received pure exercise interventions and a cognitive test. Although there was heterogeneity in intervention methods and cognitive measures among studies, meta-analysis (seven studies) supports positive effects of physical activity on cognitive function of patients with $\mathrm{AD}$ (mean difference $[\mathrm{MD}]=2.53$, the $95 \% \mathrm{CI}=0.84$ to 4.22 , test for overall effect: $Z=2.93$ $[P=0.003])$. Eight studies demonstrated that exercise improves cognitive function for individuals with AD. However, the remaining five studies did not display a beneficial effect of exercise on cognitive function in patients with AD.

Conclusion: This meta-analysis and systematic review indicated that exercise intervention might improve the cognitive function of $\mathrm{AD}$ or slow down the decline of cognition; however, this relationship was not always true across studies. RCTs with clear intervention criteria, large samples, and long-term follow-up are needed in the future to demonstrate the benefits of exercise for cognitive function in $\mathrm{AD}$ patients.

Keywords: Alzheimer's disease, exercise, cognitive function, randomized controlled trial

\section{Introduction}

Alzheimer's disease (AD) is an insidious and progressive neurodegenerative disease characterized by cognitive dysfunction. It has a high prevalence, ${ }^{1}$ and there exists no effective treatment, resulting in a burden to family and society. AD is the most common form of dementia, accounting for $60 \%-70 \%$ of patients with dementia. ${ }^{2}$ Dementia is a chronic and progressive neurological disease, which is clinically characterized by the slow onset of memory deficits, accompanied by varying degrees of personality changes. It is a group of clinical syndromes rather than an independent disease. Currently, there are $\sim 47$ million people with dementia worldwide, ${ }^{3}$ and the number is expected to triple by $2050 .{ }^{4}$ Dementia significantly erodes the function and 
quality of life, impedes economic and social development, and brings burdens and pressures to families. Cost associated with dementia exceed that of heart disease and cancer and is usually paid directly by the family. ${ }^{5}$ Other types of dementia include frontal-temporal dementia, dementia with Lewy bodies, Parkinson's disease, Huntington's disease, and vascular dementia. Treatment of dementia should be directed toward the root cause of the disease. However, there currently exists no specific drug for the treatment of neurodegenerative diseases. Treatment is primarily aimed at improving cognition and relieving symptoms. Traditional treatments are mainly pharmacotherapy; however, pharmacotherapies only relieve symptoms and might have side effects. ${ }^{6}$ Exercise can be used as an adjuvant therapy before effective new drugs are developed and has thus emerged as a treatment option. An update of the 2001 American Academy of Neurology of guidelines on mild cognitive impairment (MCI) demonstrated that exercise training (6 months) might improve cognitive function in MCI patients. ${ }^{7}$ Several studies have demonstrated that physical exercise can slow down the progression of cognitive declines. Studies have demonstrated that exercise reduces the risk of dementia in older adults who are cognitively normal. ${ }^{8,9}$ Kemoun et al ${ }^{10}$ conducted a 15-week physical activity program and demonstrated that subjects in the intervention group (IG) had improved overall Rapid Evaluation of Cognitive Function (ERFC) scores $(P<0.01)$, while the control group (CG) ERFC scores declined. Yang et $\mathrm{al}^{11}$ conducted a 3-month randomized controlled trial (RCT) with 50 patients with $\mathrm{AD}$, and they demonstrated that moderate intensity aerobic exercise can improve cognitive function in patients with AD. Yágüez et al ${ }^{12}$ carried out a study which indicated that a short course of nonaerobic movement-based exercise is effective at improving some aspects of cognitive function in patients with $\mathrm{AD}$. In addition, many systematic reviews and meta-analyses show a positive effect of physical activity as an intervention therapy. A systematic review by Farina et $\mathrm{al}^{13}$ demonstrated that exercise generally had a positive effect on the rate of cognitive decline in AD. Similarly, a meta-analysis by Heyn et $\mathrm{al}^{14}$ involving 2,020 participants demonstrated that exercise training increased cognitive function in people with dementia and related cognitive impairments. Scherder et a ${ }^{15}$ carried out a systematic review and meta-analysis, which indicated a positive finding that participants leading a sedentary life might prevent or delay declines in executive function when following a walking program. However, these conclusions are not always observed, most studies vary in terms of research design, nonstandardized interventions, and research type. Although studies that emphasize the importance of exercise exist, the effects of exercise on cognitive functions in Alzheimer's patients are mixed as some studies have observed no positive effect of physical activity on cognitive function in people with AD. An updated review in 2013 found no evidence of cognitive benefits in patients with AD. ${ }^{16} \mathrm{~A}$ systematic review of Littbrand et $\mathrm{al}^{17}$ demonstrated that whether physical exercise can improve cognitive functions among people with dementia remains unclear. Öhman et al ${ }^{18}$ conducted a systematic review of RCTs on the cognition of older patients with dementia and found that most studies demonstrated that exercise has no effect on the cognitive function.

Stronger evidence is needed to confirm the role of exercise in cognitive function in patients with AD. The purpose of this meta-analysis and systematic review was to review research that demonstrates the efficacy of exercise on cognitive function of AD. Furthermore, in order to reduce the heterogeneity among studies resulting from inherent study differences, we included only RCTs for analysis, to gain a greater understanding of this relationship and to provide a more informed suggestion by analyzing the evidence.

\section{Materials and methods Study selection strategy}

The bibliographic databases (PubMed, Cochrane Library and Embase, and Web of Science) and four Chinese databases (Wanfang data, CBM, CNKI, and VIP) were searched to identify RCTs published in any language between January 1, 1960, and January 1, 2018. At first, Medical Subject Headings (MeSH) of exercise were identified for $\mathrm{AD}$ and cognition by searching the Chinese Medical Subject Headings (CMeSH) on SinoMed. This was followed by using "MeSH" to identify individual entry terms that were used as keywords in subsequent searches. Finally, we retrieved articles using the search builder of MeSH terms and various combinations of the entry terms "exercise(s)", "physical activity", "isometric exercise", "resistance training", "aerobic exercise", "running", "jogging", "swimming", "walking”, "stair climbing”, "Muscle Stretching Exercises", "yoga", "tai chi", with AD terms "Alzheimer('s) disease" or "dementia, Alzheimer type" or "Alzheimer syndrome" or "AD", as well as the cognition terms "cognition(s)" or "cognitive function(s)". (see Table S1 for search strategies in detail) Only peer-reviewed articles and RCTs were included, and animal studies were excluded. More relevant studies were retrieved from the meta-analysis and the list of references of related studies. 
We used citation search to check the results of the search by searching relevant systematic reviews.

\section{Study design}

Based on the following criteria, we selected eligible studies for our meta-analysis from the initial search: 1) participants: trials enrolled patients with clinically diagnosed AD. There is no limit to the severity of $\mathrm{AD}$. We excluded studies that included patients who might have a disease that affects cognitive function (such as stroke, cancer, Parkinson's disease, and traumatic brain injury). Participants with Lewy body dementia, frontotemporal dementia, vascular dementia, or other rare forms of dementia were also excluded; 2) interventions: the research must have physical exercise intervention, such as aerobic fitness, strength training, balance and flexibility training, cycling training, walking, and stamina. Trials of multimodal interventions such as exercise accompanied by music therapy or cognitive therapy were excluded. There was no restriction on the type, intensity, and frequency of physical activity; 3) outcome measures: the trial must have a measured cognitive outcome for patients with $\mathrm{AD}$, including the use of scales or other cognitive measurement tools; and 4) the study must be an RCT.

\section{Data extraction and quality assessment}

Two investigators independently reviewed the title and abstract of search results and screened the full text of references that might be eligible. When differences occurred in eligibility of inclusion, exclusion, or data extraction, a third reviewer participated in the discussion. Physical activity interventions of all sample sizes were included. Author, publication year, country, age, sample size, intervention characteristics, duration, measurement instrument, results, and dropouts were included for all eligible studies. Two researchers assessed the risk of bias independently using the Cochrane handbook (5.1.0). ${ }^{19}$

\section{Quantitative data synthesis}

Review Manager (5.3) was used to estimate the overall effect of physical therapy in each study. The intervention effect was described as mean $\pm \mathrm{SD}$ with $95 \% \mathrm{CI}$ of cognitive scores of postintervention between the IG and CG, respectively. According to the Cochrane Collaboration handbook for systematic reviews of interventions, selection of fixed or random effects meta-analysis should be based on the potential real effect of intervention on outcome measures. ${ }^{19}$ Considering the heterogeneity of intervention type, result measurement, and sample characteristics, we chose to use a random effects model as a matter of priority. ${ }^{20}$ Statistical heterogeneity was revealed by $I^{2}$ statistics. When $I^{2}$ is $>50 \%$, the result is considered heterogeneous. ${ }^{21}$ In addition, it is said that because the random effects model does not provide a quantitative measure of heterogeneity, it was evaluated by visual inspection of the funnel plot. ${ }^{22}$ Therefore, both methods were used to assess heterogeneity. $P$-value $<0.05$ was considered statistically significant.

\section{Results}

Initially, 3,327 articles were obtained through database search, and 16 articles were retrieved by performing manual searches of the selected key references. After removing duplicates, there were 2,398 articles remaining. Furthermore, 1,855 trials were excluded as they covered an irrelevant topic. After reviewing the title and abstract, 530 trials were excluded due to the following reasons: insufficient or irrelevant outcome information ( $\mathrm{n}=356)$, study designs $(\mathrm{n}=57)$, multi-modal interventions $(\mathrm{n}=9)$, inclusion of nondemented patients $(\mathrm{n}=106)$, and unlocated full text $(\mathrm{n}=2)$. As a result of the screening process, $13 \mathrm{RCT}$ articles were selected, ${ }^{10-12,23-32}$ which included 659 patients who were diagnosed with $\mathrm{AD}$ as presented in Figure 1. Patients were split into control (319) or patients receiving intervention treatment (340). Sample sizes of studies ranged from 21 to 200 , and studies were published between 2002 and 2017. Cognitive result measurements usually included Mini-Mental State Examination (MMSE) (nine studies) $)^{11,23-30}$ or other cognitive function tests such as Alzheimer's Disease Assessment Scale - Cognitive SubScale, ${ }^{27-29}$ verbal fluency, ${ }^{29}$ clock-drawing test, ${ }^{30}$ ERFC, ${ }^{10}$ Cambridge Neuropsychological Test Automated BatteryExpedio, ${ }^{12}$ test of phonemic verbal fluency, ${ }^{24}$ Stroop Color and Word Test, ${ }^{29}$ Frontal Assessment Battery, ${ }^{30}$ and Functional Assessment of Communication Skills Mental Subscale. ${ }^{31}$ More information or characteristics of the included studies are summarized in Table 1. An assessment of bias in each field is presented in Figures 2 and 3. A meta-analysis was conducted on seven articles that had available data for MMSE as an observation index, and a comparison of the experimental group and the CG is presented in Figure 4. The heterogeneity of the included articles is shown in Figure 5.

\section{Risk of bias assessment}

In order to avoid false findings in the comprehensive analysis and to ensure reliability of results, quality of selected studies was assessed for risk of bias. The risk of bias graph and the authors' judgment about each risk of bias item are presented in Figure 2. Figure 2 indicates the quality details 


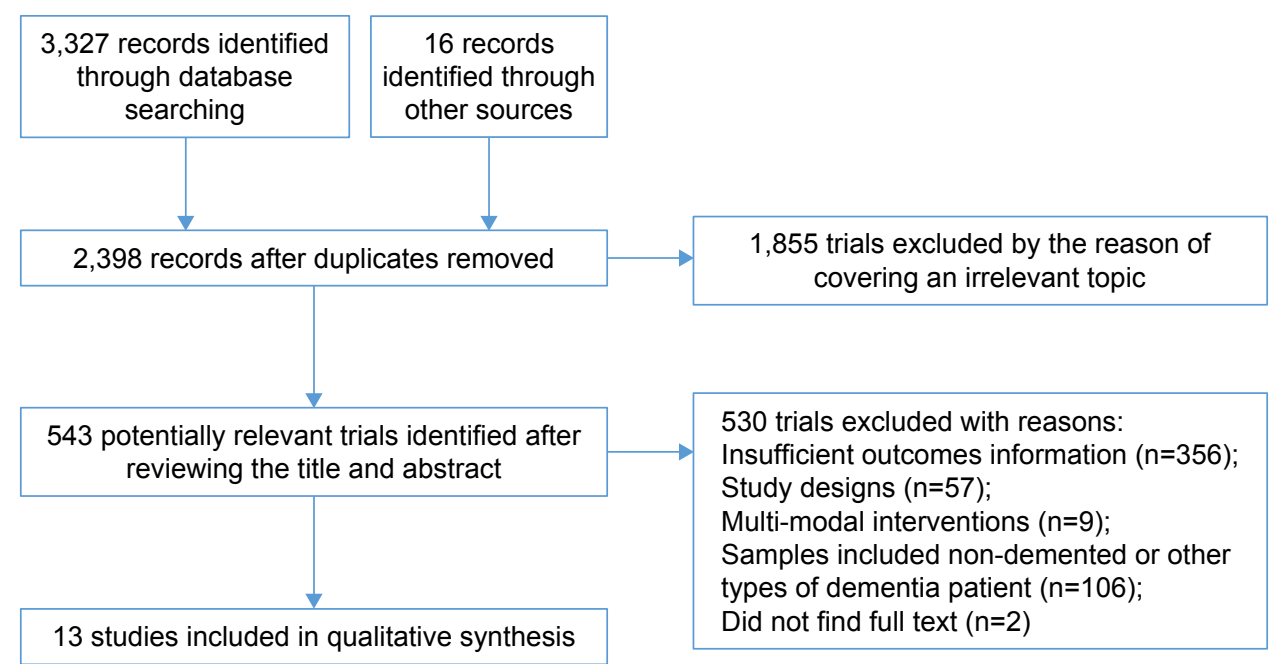

Figure I Flow diagram of the study identification process.

of each study and demonstrates that only one research has a high-risk quality assessment. ${ }^{31}$ In one trial, individuals had a different baseline in MMSE. ${ }^{25}$ In addition, the study carried out by Cott et $\mathrm{al}^{31}$ had a high risk for incomplete outcome data (attrition bias) and considerable individual variability in MMSE scores, and it did not use intervention-to- to treat to avoid the occurrence of bias. The authors' judgments of each risk of bias item are presented in Figure 3. In general, the proportion of high-risk bias is small.

\section{Meta-analysis of the seven studies supports exercise as a treatment option to help improve cognition of AD patients}

In the meta-analysis of the seven articles, ${ }^{11,23,24,26-29}$ results demonstrated that studies were heterogeneous $(P<0.001$, $\left.I^{2}=89 \%\right)$, therefore a random effects model was chosen for analysis. Figure 4 demonstrates that exercise can improve cognition of $\mathrm{AD}$ patients and that total outcome: $\mathrm{MD}=2.53$, $95 \% \mathrm{CI}=0.84$ to 4.22 , and $Z=2.93$ ( $P=0.003$ ). Results of five of the included articles are consistent with overall results of this meta-analysis and support exercise as a treatment option in improving the cognition of patients with $\mathrm{AD}$. $11,23,24,26,27$ Although results from two articles do not support the findings of this study, the $95 \%$ CI of both studies covered the invalid vertical line, suggesting that the study was not statistically significant. ${ }^{28,29}$ The risk of bias of this meta-analysis is presented in Figure 5. Scatter points of each study are distributed at the top of the funnel and are symmetrically distributed, suggesting a small bias of those research studies (Figure 5).

\section{Review - complete data set $(n=\mid 3)$}

Six articles had no associated MMSE data and were not included in the meta-analysis. Eight articles demonstrated that exercise improves cognitive function for individuals with $\mathrm{AD}$ and supports physical activity as a treatment option for patients with AD. ${ }^{10-12,23,24,26,27,30}$ Kemoun et al ${ }^{10}$ found that physical activity programs slow cognitive decline: postintervention cognitive scores: intervention: $30.4 \pm 7.7$, control: 23.2 \pm 8.4 ; overall ERFC score of the subjects from the IG improved $(P<0.01)$, while that of the CG decreased. Yang et $\mathrm{a}^{11}$ recruited 50 elderly people with mild $\mathrm{AD}$ and found that MMSE score significantly increased $(P<0.05)$ in aerobic groups before and after 3 months, while for the CG, MMSE scores decreased significantly after 3 months $(P<0.05)$. These results provide strong evidence that aerobic exercise can improve cognition of $\mathrm{AD}$ patients. ${ }^{11}$ Yágüez et $\mathrm{al}^{12}$ demonstrated significant improvements in sustained attention, visual memory, and working memory in the exercise group when compared with the CG after a 6-week training regime. Kwak et al's ${ }^{23}$ findings demonstrated that MMSE score increases $20 \%$ (pre: $14.53 \pm 5.34, P<0.05$ ) and $30 \%$ (post: $17.47 \pm 6.90, P<0.01$ ) at 6 and 12 months. Venturelli et al ${ }^{26}$ aimed to compare the effect of a walking program on cognitive decline of elderly nursing home residents in the later stages of AD. They found that the walking group did not show a significant change in MMSE scores before and after the training period, whereas the CG group showed a decrease in MMSE scores during the same period. Vreugdenhil et $\mathrm{al}^{27}$ examined the effect of community-based exercise programs on AD patients. After 4 months, patients who exercised had improved cognition when compared with 


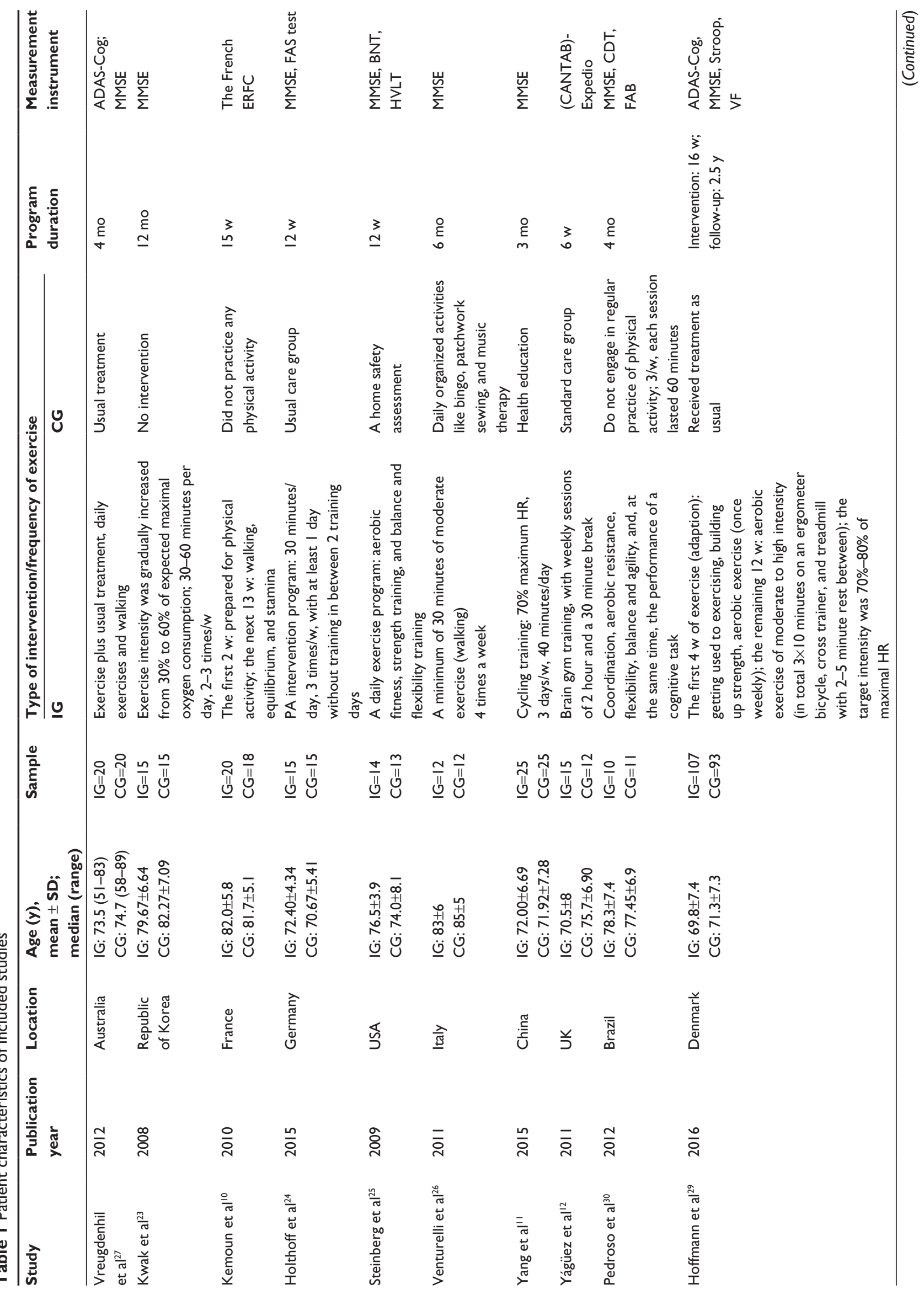




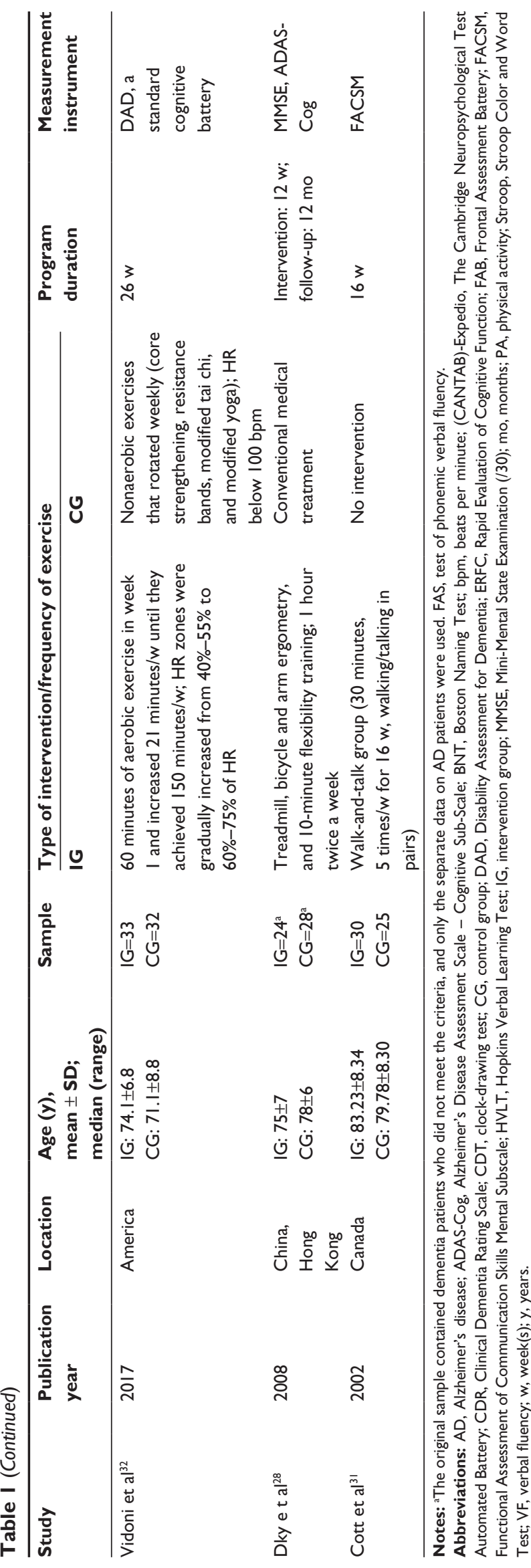

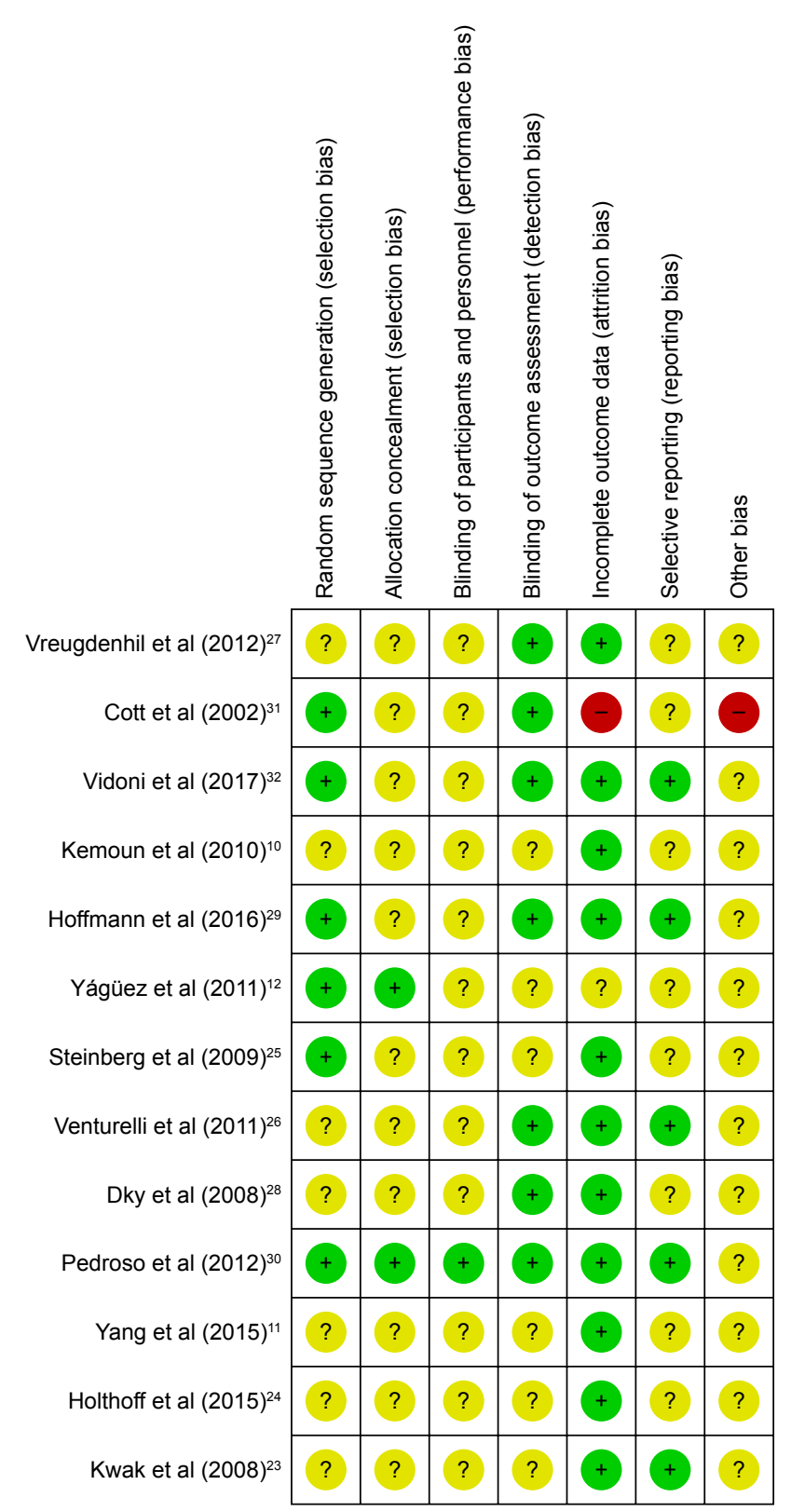

Figure 2 Risk of bias summary: review authors' judgments about each risk of bias item for each included study.

control (increased MMSE scores by 2.6 points, $P<0.001$ ). Pedroso et $\mathrm{al}^{30}$ studied 21 older patients with probable AD and detected a positive interaction between groups and moments $(P=0.000479)$, confirming the effect of physical activity on global cognitive function. In addition, the outcomes of MMSE, FAB, and CDT of IG were all positive with $P<0.05 .{ }^{30}$ However, the remaining five studies did not display a beneficial effect of exercise on cognitive function in patients with $\mathrm{AD} \cdot{ }^{25,28,29,31,32}$ It is important to note that one study observed that the effects of exercise on cognitive function in patients with $\mathrm{AD}$ differed from each other when different observational indicators were used..$^{32}$ 


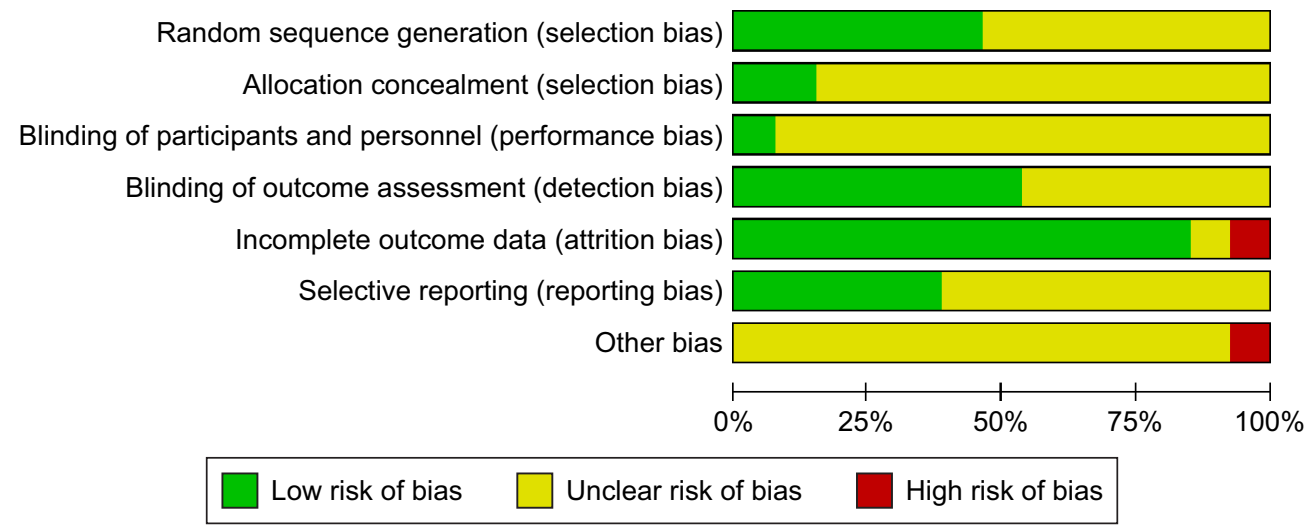

Figure 3 Risk of bias graph: review authors' judgments about each risk of bias item presented as percentages across all included studies.

\section{Discussion}

The prevalence of $\mathrm{AD}$ has increased over recent years, resulting in a heavy burden to families, economies, and society. At present, there are no effective therapeutic drugs. Exercise is a preferred nonpharmacotherapy treatment option due to its wide range of positive effects, fewer side effects, and low economic burden. However, to date, results of studies have been inconsistent regarding the effectiveness of exercise in improving cognitive function in $\mathrm{AD}$ patients. Therefore, literature was systematically reviewed to investigate the effect of exercise on $\mathrm{AD}$ patients. Initially, 3,343 articles were retrieved; however, as a result of the screening process, 13 articles were selected. This study presents evidence supporting exercise as an effective intervention option for cognitive function in patients with $\mathrm{AD}$, although the methodological heterogeneity in the literature limits our conclusion. Eight studies demonstrated that exercise has a positive effect on cognitive function in people with $\mathrm{AD} \cdot{ }^{10-12,23,24,26,27,30}$ The remaining articles had mixed results or did not demonstrate statistical differences. ${ }^{25,28,29,31,32}$ Additionally, types of physical activity are different. Nine studies had an intervention period between 12 and 16 weeks, ${ }^{10,11,24,25,27-31}$ while other trials had an intervention period from 1.5 to 12 months. ${ }^{12,23,26,32}$ In eight studies, the CG had patients with no exercise intervention or treatment as usual, ${ }^{10,12,23,24,27-29,31}$ in one trial patients in the CG performed nonaerobic exercises, ${ }^{32}$ and in one trial, patients in the CG participated in talk only visits..$^{31}$ It is important to note that selected studies used several cognitive tests, and MMSE was the most commonly used cognition detection method. Nine studies used MMSE as a measure of cognitive ability, ${ }^{11,23-30}$ nine studies applied various other monitoring indicators, ${ }^{10,12,24,25,27-31}$ and seven studies identified cognition by means of multiple outcome measures. ${ }^{24,25,27-30,32}$ One study presented inconsistent results in different outcome measures for the same study. ${ }^{32}$ It is therefore difficult to make direct comparisons. In addition, it was hard to determine whether exercise is invalid or the cognitive measurement tools are not properly selected when there are no significant differences between the two groups. Because the selected studies demonstrated great heterogeneity in type of intervention,

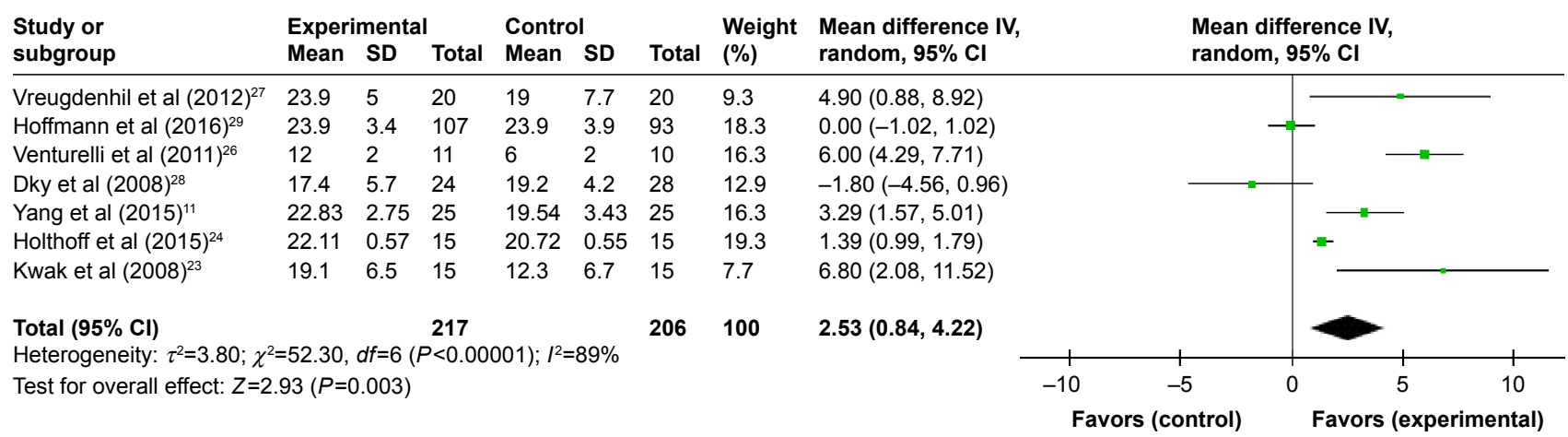

Figure $4 \mathrm{~A}$ forest plot shows the results of a meta-analysis of six RCTs measuring MMSE outcome for individuals with AD.

Note: Exercise intervention had a positive effect on MMSE results.

Abbreviations: AD, Alzheimer's disease; MMSE, Mini-Mental State Examination; RCT, randomized controlled trial. 


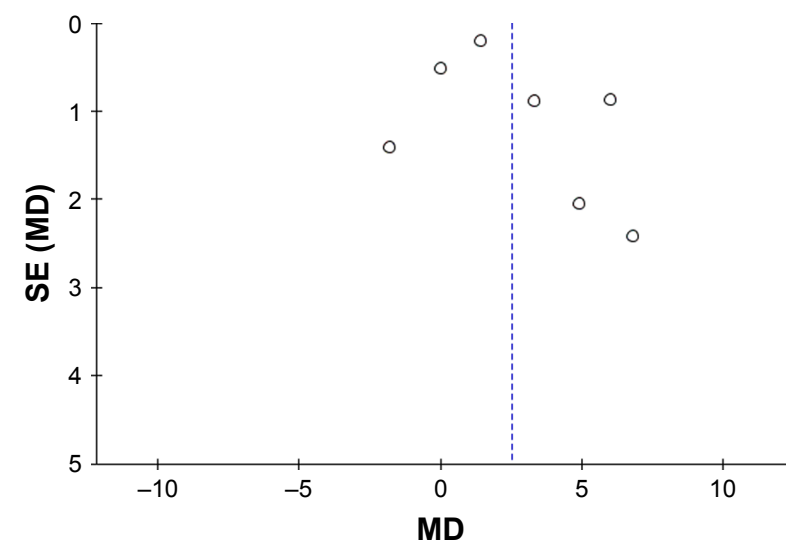

Figure 5 A funnel plot of the meta-analysis of six studies.

Notes: SE indicates the accuracy of the estimated value of the treatment effect, and the smaller the SE, the more accurate it is. The MD measures the treatment effect size.

Abbreviations: MD, mean difference; SE, standard error.

duration, intensity, and frequency of exercise, there is a need to develop uniform research criteria, including the characteristics of the participants, research designs, intervention methods, selection of cognition measurements, and so on, in order to conduct large sample studies with uniform standards, which can provide a reliable basis for clinical treatment application.

It is difficult to achieve high-frequency and continuous intervention for $\mathrm{AD}$ patients in real-world studies. In addition, it is difficult to perform exercise-intervened RCT in $\mathrm{AD}$ patients. These results are inconsistent and controversial. Exercise conditions are usually self-reported data with uncertain accuracy, and it is difficult to exclude the impact of other factors, such as diet, environment, social support, and so on, on the results of exercise intervention; for the elderly, especially those with moderate to severe dementia, exercise intervention is potentially dangerous. Therefore, exercise interventions have limitations. Well-designed research is needed to solve these problems in the future. Due to the inconsistency in monitoring indicators, meta-analysis can only be conducted on studies with MMSE as an outcome measure. Due to the unavailability of MMSE outcome data for two studies, ${ }^{25,30}$ this study conducted a meta-analysis of seven studies. ${ }^{11,23,24,26-29}$ Overall, results of this meta-analysis support exercise to improve cognitive function in people with $\mathrm{AD}$, which is in line with our expectation. In the quantitative meta-analysis, research types and result measurement tools included are the same, greatly improving the strength of the evidence and adding much more authority to the evidence. Of the six studies that were not included in this meta-analysis, although three studies failed to achieve positive results, lack of results did not mean that exercise was not effective. In one study, no significant differences in the outcomes measured posttest were found. In this study, participants in the CG received home safety training. ${ }^{25}$ In one study, patients in the CG participated in talk only visits. ${ }^{31}$ In one study, participants in the CG were asked to do stretching and toning, and the Disability Assessment for Dementia total functional independence was associated with changes in memory-specific cognitive function $(r=0.26,95 \% \mathrm{CI}=0.06$ to $\infty, P=.017)$ but not executive function $(r=0.18,95 \% \mathrm{CI}=-0.03$ to $\infty, P=.07) .{ }^{32}$ Possible explanations for the lack of cognitive benefits in exercise include the following: 1) training activities and social participation in the CG might have produced cognitive benefits; 2) the short period of intervention might not be enough to produce cognitive benefits; and 3) the level (intervention type, time, intensity, and frequency) of physical activity might not be sufficient to produce cognitive benefits. Therefore, we believe that physical activity can improve the cognitive function of patients with $\mathrm{AD}$ and that some studies have not had long enough times of intervention or sufficient power to demonstrate the effectiveness of exercise. In order to achieve this positive result, it is necessary to start the exercise intervention in the early stage and to persevere in the long run as a daily habit.

There are three mechanisms for exercise to improve cognition. First, animal experiments demonstrated that exercise can promote neural plasticity ${ }^{33,34}$ and induce increases in neurogenesis in the hippocampus, and ${ }^{35,36}$ aerobic fitness promotes blood flow, glucose utilization, and oxygen extraction and improves functional and structural brain reserves. ${ }^{37}$ Second, exercise can promote the secretion of brain-derived neurotrophic factor (BDNF), ${ }^{38-41}$ which is related to learning and memory. Third, the indirect effects of exercise on the brain cannot be ignored. Exercise can reduce certain chronic diseases (such as cardiovascular diseases, obesity, diabetes, and so on) and the risk factors that have been associated with dementia and other cognitive dysfunctions. ${ }^{42}$

This study only included RCTs, which unified the study types, reduced bias, and provided strong evidence. In addition, the meta-analysis included seven new RCTs. The overall outcome demonstrates that physical activity benefits the cognitive function of $\mathrm{AD}$ patients. Although strength of validity evidence was not significant, physical activity as a positive lifestyle choice can improve patient's cognitive function, reduce risk of multiple diseases, and improve patients' physical function. Moreover, physical activity has less side effects and less economic burden, which has more extensive benefits than drug therapy. 
Our review also has several limitations. Although there are no language restrictions in the search strategy, the studies eventually included were in English and might have had language bias. Most trials have small sample sizes, and the intervention methods such as type of intervention, intensity, duration, and frequency are heterogeneous. The follow-up period was not long enough and measures of cognitive function were different, while some trials used several cognitive measurements. In this study, although some results were not statistically different, it is difficult to determine whether the power of exercise intervention is insufficient or the exercise is ineffective. The research protocol could not always be found; therefore, there might be some publication bias.

\section{Conclusion}

The purpose of this review was to reveal the effects of physical activity on cognitive function in patients with AD. RCTs of exercise and cognitive function in patients with $\mathrm{AD}$ were reviewed, and a positive effect was found through meta-analysis and some other research outcomes. However, the heterogeneity of intervention type, time, intensity, frequency of physical activity, cognitive tests, follow-up time, control conditions as well as small sample trials do not allow us to make a very definitive conclusion about the effects of exercise intervention. In clinical practice, it is strongly recommended to use exercise therapy to prevent or control cardiovascular diseases, obesity, and so on. Therefore, physical activity should continue to be encouraged. Targeted multimodal interventions may be more effective for different treatment goals. In addition, future trials call for standardized intervention characteristics and large-scale, long-term follow-up to generate positive outcomes that support the ability of exercise to improve cognition of $\mathrm{AD}$ patients.

\section{Acknowledgment}

This work was supported by the National Natural Science Foundation of China (number 81570491).

\section{Disclosure}

The authors report no conflicts of interest in this work.

\section{References}

1. Alzheimer's Association. 2015 Alzheimer's disease facts and figures. Alzheimers Dement. 2015;11(3):332-384.

2. Reitz C, Brayne C, Mayeux R. Epidemiology of Alzheimer disease. Nat Rev Neurol. 2011;7(3):137-152.

3. Prince M, Ali GC, Guerchet M, Prina AM, Albanese E, Wu YT, Yt W. Recent global trends in the prevalence and incidence of dementia, and survival with dementia. Alzheimers Res Ther. 2016;8(1):23.
4. Prince M, Bryce R, Albanese E, Wimo A, Ribeiro W, Ferri CP. The global prevalence of dementia: a systematic review and meta-analysis. Alzheimers Dement. 2013;9(1):63-75.

5. Kelley AS, Mcgarry K, Gorges R, Skinner JS. The burden of health care costs for patients with dementia in the last 5 years of life. Ann Intern Med. 2015;163(10):729-736.

6. Jiang T, Yu JT, Tan L. Novel disease-modifying therapies for Alzheimer's disease. J Alzheimers Dis. 2012;31(3):475-492.

7. Petersen RC, Lopez O, Armstrong MJ, et al. Practice guideline update summary: Mild cognitive impairment: Report of the Guideline Development, Dissemination, and Implementation Subcommittee of the American Academy of Neurology. Neurology. 2018;90(3):126-135.

8. Abbott RD, White LR, Ross GW, Masaki KH, Curb JD, Petrovitch H. Walking and dementia in physically capable elderly men. JAMA. 2004;292(12):1447-1453.

9. Buchman AS, Boyle PA, Yu L, Shah RC, Wilson RS, Bennett DA. Total daily physical activity and the risk of AD and cognitive decline in older adults. Neurology. 2012;78(17):1323-1329.

10. Kemoun G, Thibaud M, Roumagne N, et al. Effects of a physical training programme on cognitive function and walking efficiency in elderly persons with dementia. Dement Geriatr Cogn Disord. 2010;29(2):109-114.

11. Yang SY, Shan CL, Qing H, et al. The effects of aerobic exercise on cognitive function of Alzheimer's disease patients. CNS Neurol Disord Drug Targets. 2015;14(10):1292-1297.

12. Yágüez L, Shaw KN, Morris R, Matthews D. The effects on cognitive functions of a movement-based intervention in patients with Alzheimer's type dementia: a pilot study. Int J Geriatr Psychiatry. 2011;26(2):173-181.

13. Farina N, Rusted J, Tabet N. The effect of exercise interventions on cognitive outcome in Alzheimer's disease: a systematic review. Int Psychogeriatr. 2014;26(1):9-18.

14. Heyn P, Abreu BC, Ottenbacher KJ. The effects of exercise training on elderly persons with cognitive impairment and dementia: a metaanalysis. Arch Phys Med Rehabil. 2004;85(10):1694-1704.

15. Scherder E, Scherder R, Verburgh L, et al. Executive functions of sedentary elderly may benefit from walking: a systematic review and meta-analysis. Am J Geriatr Psychiatry. 2014;22(8):782-791.

16. Forbes D, Forbes SC, Blake CM, Thiessen EJ, Forbes S. Exercise programs for people with dementia. Cochrane Database Syst Rev. 2015(4):CD006489.

17. Littbrand H, Stenvall M, Rosendahl E. Applicability and effects of physical exercise on physical and cognitive functions and activities of daily living among people with dementia: a systematic review. Am J Phys Med Rehabil. 2011;90(6):495-518.

18. Öhman H, Savikko N, Strandberg TE, Pitkälä KH. Effect of physical exercise on cognitive performance in older adults with mild cognitive impairment or dementia: a systematic review. Dement GeriatrCogn Disord. 2014;38:347-365.

19. Higgins JPT, Green S, editors. Cochrane Handbook for Systematic Reviews of Interventions Version 5.1. 0 [updated March 2011]. London: The Cochrane Collaboration; 2011.

20. Field AP, Gillett R. How to do a meta-analysis. Br J Math Stat Psychol. 2010;63(Pt 3):665-694.

21. Higgins JP, Thompson SG. Quantifying heterogeneity in a metaanalysis. Stat Med. 2002;21(11):1539-1558.

22. Egger M, Davey Smith G, Schneider M, Minder C. Bias in meta-analysis detected by a simple, graphical test. BMJ. 1997;315(7109):629-634.

23. Kwak YS, Um SY, Son TG, Kim DJ. Effect of regular exercise on senile dementia patients. Int J Sports Med. 2008;29(6):471-474.

24. Holthoff VA, Marschner K, Scharf M, et al. Effects of physical activity training in patients with Alzheimer's dementia: results of a pilot RCT study. PLoS One. 2015;10(4):e0121478.

25. Steinberg M, Leoutsakos JM, Podewils LJ, Lyketsos CG. Evaluation of a home-based exercise program in the treatment of Alzheimer's disease: the maximizing independence in dementia (MIND) study. Int J Geriatr Psychiatry. 2009;24(7):680-685. 
26. Venturelli M, Scarsini R, Schena F. Six-month walking program changes cognitive and ADL performance in patients with Alzheimer. Am J Alzheimers Dis Other Demen. 2011;26(5):381-388.

27. Vreugdenhil A, Cannell J, Davies A, Razay G. A community-based exercise programme to improve functional ability in people with Alzheimer's disease: a randomized controlled trial. Scand J Caring Sci. 2012;26(1):12-19.

28. Dky M, Szeto SL, Mak YF. A randomised controlled trial on the effect of exercise on physical, cognitive and affective function in dementia subjects. Asian J Gerontol Geriatr. 2008;3(1):8-16.

29. Hoffmann K, Sobol NA, Frederiksen KS, et al. Moderate-to-high intensity physical exercise in patients with Alzheimer's disease: a randomized controlled trial. J Alzheimers Dis. 2016;50(2):443-453.

30. Pedroso RV, Coelho FG, Santos-Galduróz RF, Costa JL, Gobbi S, Stella F. Balance, executive functions and falls in elderly with Alzheimer's disease (AD): a longitudinal study. Arch Gerontol Geriatr. 2012;54(2):348-351.

31. Cott CA, Dawson P, Sidani S, Wells D. The effects of a walking/ talking program on communication, ambulation, and functional status in residents with Alzheimer disease. Alzheimer Dis Assoc Disord. 2002;16(2):81-87.

32. Vidoni ED, Perales J, Alshehri M, Giles AM, Siengsukon CF, Burns JM. Aerobic exercise sustains performance of instrumental activities of daily living in early-stage Alzheimer disease. $J$ Geriatr Phys Ther. 2017:1.

33. Scoville WB, Milner B. Loss of recent memory after bilateral hippocampal lesions. J Neurol Neurosurg Psychiatry. 1957;20(1):11-21.
34. O’Keefe J, Nadel L, Keightley S, Kill D. Fornix lesions selectively abolish place learning in the rat. Exp Neurol. 1975;48(1):152-166.

35. Sahay A, Scobie KN, Hill AS, et al. Increasing adult hippocampal neurogenesis is sufficient to improve pattern separation. Nature. 2011; 472(7344):466-470.

36. Swain RA, Harris AB, Wiener EC, et al. Prolonged exercise induces angiogenesis and increases cerebral blood volume in primary motor cortex of the rat. Neuroscience. 2003;117(4):1037-1046.

37. Cotman CW, Berchtold NC. Exercise: a behavioral intervention to enhance brain health and plasticity. Trends Neurosci. 2002;25(6): 295-301.

38. Belarbi K, Burnouf S, Fernandez-Gomez FJ, et al. Beneficial effects of exercise in a transgenic mouse model of Alzheimer's disease-like Tau pathology. Neurobiol Dis. 2011;43(2):486-494.

39. Nichol K, Deeny SP, Seif J, Camaclang K, Cotman CW. Exercise improves cognition and hippocampal plasticity in APOE epsilon4 mice. Alzheimers Dement. 2009;5(4):287-294.

40. Cotman CW, Berchtold NC. Physical activity and the maintenance of cognition: learning from animal models. Alzheimers Dement. 2007; 3(2 Suppl):S30-S37.

41. Adlard PA, Perreau VM, Pop V, Cotman CW. Voluntary exercise decreases amyloid load in a transgenic model of Alzheimer's disease. J Neurosci. 2005;25(17):4217-4221.

42. Kalmijn S, Foley D, White L, et al. Metabolic cardiovascular syndrome and risk of dementia in Japanese-American elderly men. The Honolulu-Asia aging study. Arterioscler Thromb Vasc Biol. 2000; 20(10):2255-2260. 


\section{Supplementary material}

Table SI The complete bibliographic strategy for PubMed

\begin{tabular}{|c|c|c|}
\hline Search & Query & $\begin{array}{l}\text { Items } \\
\text { found }\end{array}$ \\
\hline$\# I$ & $\begin{array}{l}\text { Search }((((((()(((()(((()(((()(((((\text { Alzheimer's Disease[Title/Abstract]) OR Dementia, Senile[Title/Abstract]) OR } \\
\text { Senile Dementia[Title/Abstract]) OR Dementia, Alzheimer Type[Title/Abstract]) OR Alzheimer Type Dementia[Title/ } \\
\text { Abstract]) OR Alzheimer-Type Dementia (ATD)[Title/Abstract]) OR Alzheimer Type Dementia (ATD)[Title/Abstract]) } \\
\text { OR Dementia, Alzheimer-Type (ATD)[Title/Abstract]) OR Alzheimer Type Senile Dementia[Title/Abstract]) OR Primary } \\
\text { Senile Degenerative Dementia[Title/Abstract]) OR Dementia, Primary Senile Degenerative[Title/Abstract]) OR Alzheimer } \\
\text { Sclerosis[Title/Abstract]) OR Sclerosis, Alzheimer[Title/Abstract]) OR Alzheimer Syndrome[Title/Abstract]) OR } \\
\text { Alzheimer Dementia[Title/Abstract]) OR Alzheimer Dementias[Title/Abstract]) OR Dementia, Alzheimer[Title/Abstract]) } \\
\text { OR Dementias, Alzheimer[Title/Abstract]) OR Senile Dementia, Alzheimer Type[Title/Abstract]) OR Acute Confusional } \\
\text { Senile Dementia[Title/Abstract]) OR Senile Dementia, Acute Confusional[Title/Abstract]) OR Dementia, Presenile[Title/ } \\
\text { Abstract]) OR Presenile Dementia[Title/Abstract]) OR Alzheimer Disease, Late Onset[Title/Abstract]) OR Late Onset } \\
\text { Alzheimer Disease[Title/Abstract]) OR Alzheimer's Disease, Focal Onset[Title/Abstract]) OR Focal Onset Alzheimer's } \\
\text { Disease[Title/Abstract]) OR Familial Alzheimer Disease (FAD)[Title/Abstract]) OR Alzheimer Disease, Familial (FAD) } \\
\text { [Title/Abstract]) OR Alzheimer Diseases, Familial (FAD)[Title/Abstract]) OR Familial Alzheimer Diseases (FAD)[Title/ } \\
\text { Abstract]) OR Alzheimer Disease, Early Onset[Title/Abstract]) OR Early Onset Alzheimer Disease[Title/Abstract]) OR } \\
\text { Presenile Alzheimer Dementia[Title/Abstract]) OR Alzheimer Disease[Title/Abstract]) OR AD[Title/Abstract] }\end{array}$ & 126045 \\
\hline$\# 2$ & Search "Alzheimer Disease"[Mesh] & 82670 \\
\hline$\# 3$ & $\# 1$ or \#2 & 164728 \\
\hline$\# 4$ & $\begin{array}{l}\text { Search Exercise[Title/Abstract] OR Exercises[Title/Abstract] OR Physical Activity[Title/Abstract] OR Activities, } \\
\text { Physical[Title/Abstract] OR Activity, Physical[Title/Abstract] OR Physical Activities[Title/Abstract] OR Exercise, } \\
\text { Physical[Title/Abstract] OR Exercises, Physical[Title/Abstract] OR Physical Exercise[Title/Abstract] OR Physical } \\
\text { Exercises[Title/Abstract] OR Acute Exercise[Title/Abstract] OR Acute Exercises[Title/Abstract] OR Exercise, } \\
\text { Acute[Title/Abstract] OR Exercises, Acute[Title/Abstract] OR Exercise, Isometric[Title/Abstract] OR Exercises, } \\
\text { Isometric[Title/Abstract] OR Isometric Exercises[Title/Abstract] OR Isometric Exercise[Title/Abstract] OR Exercise, } \\
\text { Aerobic[Title/Abstract] OR Aerobic Exercise[Title/Abstract] OR Aerobic Exercises[Title/Abstract] OR Exercises, } \\
\text { Aerobic[Title/Abstract] OR Exercise Training[Title/Abstract] OR Exercise Trainings[Title/Abstract] OR Training, } \\
\text { Exercise[Title/Abstract] OR Trainings, Exercise[Title/Abstract] OR Running[Title/Abstract] OR runnings[Title/Abstract] } \\
\text { OR jogging[Title/Abstract] OR joggings[Title/Abstract] OR Swimming[Title/Abstract] OR Walking[Title/Abstract] OR } \\
\text { Ambulation[Title/Abstract] OR Stair Climbing[Title/Abstract] OR Climbing, Stair[Title/Abstract] OR Stair Navigation[Title/ } \\
\text { Abstract] OR Navigation, Stair[Title/Abstract] OR Muscle Stretching Exercises[Title/Abstract] OR yoga[Title/Abstract] } \\
\text { OR tai chi[Title/Abstract] OR Tai-ji[Title/Abstract] OR Tai Chi[Title/Abstract] OR Chi, Tai[Title/Abstract] OR Tai } \\
\text { ji Quan[Title/Abstract] OR ji Quan, Tai[Title/Abstract] OR Quan, Tai ji[Title/Abstract] OR Taiji[Title/Abstract] } \\
\text { OR Taijiquan[Title/Abstract] OR T'ai Chi[Title/Abstract] OR Tai Chi Chuan[Title/Abstract] OR Muscle Stretching } \\
\text { Exercises[Title/Abstract] OR Exercise, Muscle Stretching[Title/Abstract] OR Exercises, Muscle Stretching[Title/Abstract] }\end{array}$ & 451829 \\
\hline$\# 5$ & Search "Exercise"[Mesh] & 165076 \\
\hline$\# 6$ & $\# 4$ or \#5 & 482666 \\
\hline$\# 7$ & $\begin{array}{l}\text { Search }(((((\text { cognition[Title/Abstract]) OR cognitions[Title/Abstract]) OR cognitive function[Title/Abstract]) OR cognition } \\
\text { functions[Title/Abstract]) OR Function, Cognitive[Title/Abstract]) OR Functions, Cognitive[Title/Abstract] }\end{array}$ & 89176 \\
\hline$\# 8$ & Search “Cognition”[Mesh] & 139349 \\
\hline$\# 9$ & $\# 7$ or \#8 & 202199 \\
\hline$\# 10$ & $\# 3$ and \#6 and \#9 & 478 \\
\hline
\end{tabular}

Notes: Sort by: most recent. Date: 20/05/2018.

Clinical Interventions in Aging

\section{Publish your work in this journal}

Clinical Interventions in Aging is an international, peer-reviewed journal focusing on evidence-based reports on the value or lack thereof of treatments intended to prevent or delay the onset of maladaptive correlates of aging in human beings. This journal is indexed on PubMed Central, MedLine,

\section{Dovepress}

CAS, Scopus and the Elsevier Bibliographic databases. The manuscript management system is completely online and includes a very quick and fair peer-review system, which is all easy to use. Visit http://www.dovepress. com/testimonials.php to read real quotes from published authors. 